\title{
Adding Structure to Statistics: A Study on COVID-19 Dynamics in India
}

\author{
Arundhati Dixit. ( Sarthak Vishnoif and Sourabh Bikas Pau周
}

(Dated: May 29, 2020)

\begin{abstract}
This study pertains to COVID-19 in India, and begins by uncovering the statistical relationship between three time series- number of cases, number of deaths, and number of tests each day, using structural vector autoregression. Further, impulse responses of the number of tests are studied, showing that doubling testing levels results in identifying $13.84 \%$ more cases. Effect of temperature is negative, and humidity is found to have no impact on number of cases, using the fixed effects model on city-level panel data. The next model utilises exponential smoothing for projecting current trend, deviation from which potentially indicates peak arrival or downturn in days to come. Lastly, multiple iterations of compartmental modelling, lockdown scenarios, indicating nearly $44.06 \%$ increase in number of cases due to untimely end of lockdown in June, the prediction being highly sensitive to model assumptions. The study quantifies concerns about under reporting, testing levels, cause for deaths, and lockdown policies.
\end{abstract}

Keywords: Time series forecasting, COVID-19, India, Regression Model, Holt Exponential Smoothing, Compartmental Model (SEIR)

\section{INTRODUCTION}

India recorded its first COVID-19 case on January 30, 2020 , and since then the numbers have steadily increased. As on May 25, 2020, a total of 144,941 cases have been reported in the country. Table II depicts COVID-19 statistics for some countries. Table I lists the same for some Indian states. Given the huge variation in reported statistics, and numbers failing to corroborate to a certain estimate of disease dynamics like death rate, we ask ourselves an important question: Do statistical anomalies arise because of under-reporting, or erroneous estimates because of the contagious and hard to trace nature of disease cause these? Other possible reasons outside our scope for these observations might be diversity in disease mutation across geographies or difference in immunity and healthcare for individuals.

This paper is motivated by the need for understanding the relationship between time series of total number of positive cases, testing done and number of deaths in India in order to know how and when does one affect the other. This produces better forecasts, and aids in healthcare, policy planning and implementation, and would help in the study of such similar epidemic study if and when needed. We utilise the concept of structural vector autoregression (SVAR) to uncover interdependence, produce short term forecasts, and look at effects of other relevant factors like increased testing facilities, and temperature and humidity. In addition to this, we anal-

\footnotetext{
* Undergraduate student at Department of Mechanical Engineering, Indian Institute of Technology Delhi arundhatidixit30@gmail.com

$\dagger$ Undergraduate student at Department of Computer Science \& Engineering, Indian Institute of Technology Delhi sarthakvishnoi01@gmail.com

¥ Faculty with Department of Humanities \& Social Sciences, Indian Institute of Technology Delhi sbpaul@hss.iitd.ac.in
}

\begin{tabular}{lllll}
\hline \hline Country & TPM & Deaths & \multicolumn{1}{c}{$\boldsymbol{\beta}_{\boldsymbol{t}}$} & $\boldsymbol{\beta}_{\boldsymbol{t}^{\mathbf{2}}}$ \\
\hline Germany & 42922 & $4.95 \%$ & $2.4 \mathrm{e}-1(1.0 \mathrm{e}-2)$ & $-1.11 \mathrm{e}-3(8.0 \mathrm{e}-5)$ \\
Italy & 57586 & $18.80 \%$ & $3.2 \mathrm{e}-1(1.0 \mathrm{e}-2)$ & $-1.8 \mathrm{e}-3(8.3 \mathrm{e}-5)$ \\
Russia & 61300 & $2.97 \%$ & $1.5 \mathrm{e}-1(1.1 \mathrm{e}-2)$ & $-1.0 \mathrm{e}-4(9.5 \mathrm{e}-5)$ \\
USA & 45521 & $17.89 \%$ & $2.3 \mathrm{e}-1(1.1 \mathrm{e}-2)$ & $-7.4 \mathrm{e}-4(8.8 \mathrm{e}-5)$ \\
India & 2200 & $6.43 \%$ & $1.4 \mathrm{e}-1(8.5 \mathrm{e}-3)$ & $-2.3 \mathrm{e}-3(7.0 \mathrm{e}-4)$ \\
\hline \hline
\end{tabular}

TABLE I. Worldwide statistics as on May 25, 2020 [4] 7]

\begin{tabular}{llllc}
\hline \hline States $/ U T$ & TPM & Deaths & \multicolumn{1}{c}{$\boldsymbol{\beta}_{\boldsymbol{t}}$} & $\boldsymbol{\beta}_{\boldsymbol{t}^{\mathbf{2}}}$ \\
\hline W Bengal & 1640 & $16.43 \%$ & $2.1 \mathrm{e}-1(5.8 \mathrm{e}-3)$ & $-1.4 \mathrm{e}-3(8 \mathrm{e}-5)$ \\
Gujarat & 2972 & $11.80 \%$ & $2.2 \mathrm{e}-1(6.4 \mathrm{e}-3)$ & $-1.5 \mathrm{e}-3(9.1 \mathrm{e}-5)$ \\
MP & 1891 & $7.75 \%$ & $2.5 \mathrm{e}-1(6.8 \mathrm{e}-3)$ & $-2.2 \mathrm{e}-3(9.9 \mathrm{e}-5)$ \\
Maharashtra & 3320 & $9.70 \%$ & $1.9 \mathrm{e}-1(2.7 \mathrm{e}-3)$ & $-1.1 \mathrm{e}-3(3.5 \mathrm{e}-5)$ \\
Delhi & 9183 & $3.92 \%$ & $2.25 \mathrm{e}-1(6.1 \mathrm{e}-3)$ & $-1.6 \mathrm{e}-1(8 \mathrm{e}-5)$ \\
\hline \hline
\end{tabular}

TABLE II. India, state-wise as on May 25, 2020 [5]

Deaths are defined as \% of no. of deaths in closed cases, and TPM is Tests Per Million. Parameter estimates are $\log$ no. of cases regressed on time $t$ and $t^{2}$, and brackets report standard errors.

yse the usefulness of exponential smoothing model for short term forecasts, and propose observational conjectures based on the same. Using relative trend of actual and forecast numbers, we propose a method for identification of having reached the peak number of cases. We strengthen short term forecasts by defining bounds using a committee of models. Lastly, we utilise iterations of long term epidemiological model, SEIR to comment on lockdown policies and its effect on long term disease dynamics. We propose a modification in the classical model which captures the current scenario.

This study tests several conjectures and adds to the existing literature on statistical analysis of COVID-19 cases. It helps produce richer predictions of number of cases in India. The study also proposes suitable modifications in using econometric methods to understand evolution of disease in the country. More details about the modelling can be found in the online appendix. 

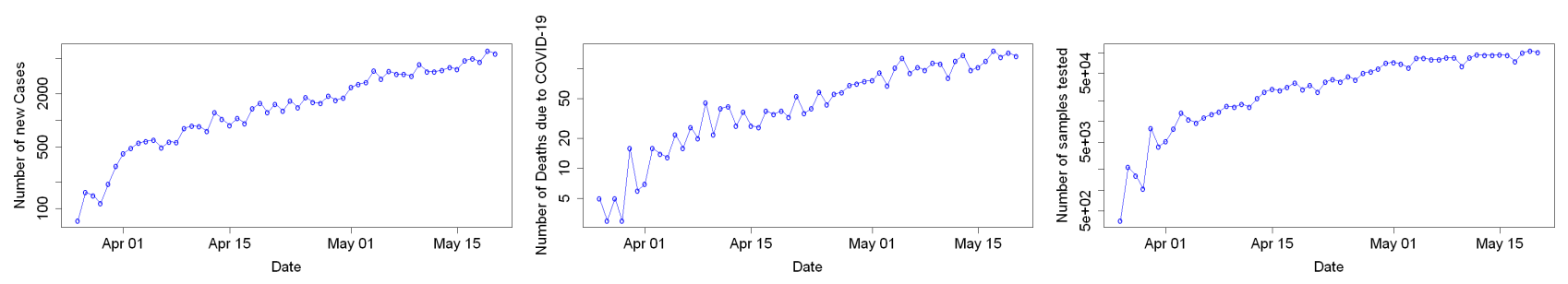

FIG. 1. The three time series, a) $x_{t}$, b) $y_{t}$, c) $z_{t}$

\section{RELATED WORK}

A number of statistical studies for COVID-19 cases have been carried out and would be underway. Regression, exponential smoothing and compartmental modelling are standard procedures for forecasting.

The study by Deb and Majumdar 2020] analyzed the number of cases per million population in various provinces of China using an ARIMA model with a structural break. Qi et al. 2020] showed the effects of Temperature and Relative Humidity on the number of confirmed cases in cities across SE Asia and China. Gupta et al. 2020 have studied the trends for the underlying time series for the number of Confirmed Cases in India for the early part of the spread (till March) in the country. The study published by Petropoulos and Makridakis [2020] utilises exponential smoothing to estimate forecasts for cumulative daily figures aggregated globally of three variables: confirmed cases, deaths and recoveries. The study by Yang et al. 2020 has used SEIR modelling with assumptions and scenario tailored to the situation in China to forecast disease dynamics. Prem et al. 2017. published a study, capturing heterogeneities in contact networks, and the effect of the same in epidemic models. Specific to India, Singh and Adhikari 2020 have studied modelling lockdown scenarios based on SIR modelling.

\section{DATA AND IMPLEMENTATION}

All the data [5, 8, compiled and condensed by us can be found on this repository. The link also has the codes that have been used to generate results presented in the submission. All the methods are implemented using RStudio version 1.2.5033, along with $\mathrm{R}$ version 3.5.1. The data used is till May 20, 2020.

\section{REGRESSION ANALYSIS}

\section{A. How are the three time series inter related?}

For India on a particular day, we consider $x_{t^{-}}$the number of confirmed COVID-19 cases; $y_{t^{-}}$number of deaths; $z_{t^{-}}$ number of samples tested; $t$ - time. Figure 1 shows the evolution of the three time series.
Intuitively, we may expect some correlation between these three time series, and use the same to define our model. For instance, an increase in confirmed cases should lead to an increase in testing in the country. Increase in testing potentially leads to an increase in COVID-19 cases contemporaneously. Increase in number of deaths due to the disease should make the authorities more proactive, and hence more testing will be done to identify the patients in the coming days. Increase in number of confirmed cases should also lead to an increase in number of deaths in the country due to COVID-19. In order to check these conjectures, we construct the model for the three time series using structural vector autoregression (SVAR). We test for stationarity of series, and conclude that all three series are trend stationary, hence during our formulation we also include time as a regressor. Models with different degrees of time variable on the basis of AIC values are compared in the paper by Deb and Majumdar, and $t$ and $t^{2}$ are found to be best suited, hence used here. Based on the PACF plots and AIC values, we include lagged values (lag $=3$ ) for the regressand. We regress on log transformation of variables.

$$
A p_{t}=\beta_{0}+\Gamma_{1} p_{t-1}+\Gamma_{2} p_{t-2}+\Gamma_{3} p_{t-3}+\beta_{1} t+\beta_{2} t^{2}+\epsilon_{t}
$$

Here, $\boldsymbol{A}, \boldsymbol{\Gamma}_{\mathbf{1}}, \boldsymbol{\Gamma}_{\mathbf{2}}$ and $\boldsymbol{\Gamma}_{\mathbf{3}}$ are $M_{3 \times 3}$ matrices. $\boldsymbol{p}_{\boldsymbol{t}}$ is $\left(\log \left(x_{t}\right), \log \left(y_{t}\right), \log \left(z_{t}\right)\right)^{\mathrm{T}}$ and $\boldsymbol{\beta}_{\boldsymbol{i}}$ s are the coefficients for time trends and the constant term.

$$
\boldsymbol{A}=\left(\begin{array}{lll}
a_{1,1} & a_{1,2} & a_{1,3} \\
a_{2,1} & a_{2,2} & a_{2,3} \\
a_{3,1} & a_{3,2} & a_{3,3}
\end{array}\right)=\left(\begin{array}{ccc}
1 & 0 & a_{1,3} \\
0 & 1 & 0 \\
a_{3,1} & 0 & 1
\end{array}\right)
$$

$\boldsymbol{A}$ determines the structure of contemporaneous relationship between the three time series. We apply some restrictions to the elements of $\boldsymbol{A}$ to compute it deterministically. The diagonal entries in $\boldsymbol{A}$ are 1 as each variable affects itself. $a_{1,2}$ determines the effect of deaths on the number of confirmed cases contemporaneously and $a_{2,1}$ the opposite of this. These two elements should be zero because there is no reason as to why one should affect the other at the same time. The same holds for $a_{2,3}$ and $a_{3,2}$ which are the contemporaneous relationship between the deaths and the samples tested. As discussed above, more testing leads to higher number of confirmed cases, hence, $a_{1,3}$ and $a_{3,1}$ cannot be zero. So we have two elements of 
the $\boldsymbol{A}$ which need to be estimated 1
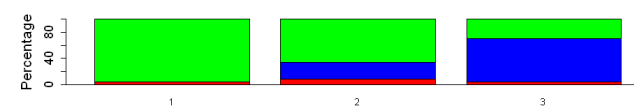

Days
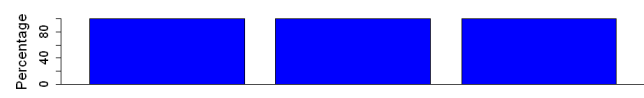

Days

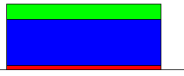

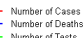
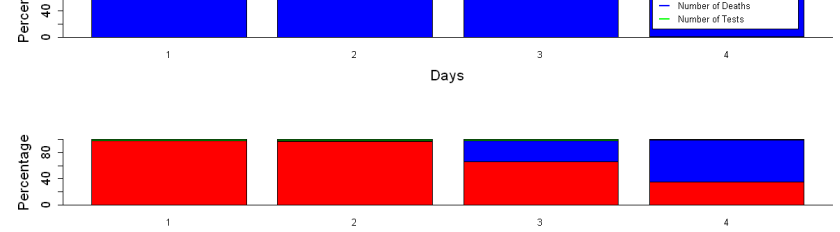

Days

FIG. 2. Forecast Error Variance Decomposition of a) $x_{t}$, b) $y_{t}$ and c) $z_{t}$

Results: Figure 2 shows the Forecast Error Variance Decomposition of the model described above. We see that a large amount of variance in errors of confirmed COVID19 cases $\left(x_{t}\right)$ comes from the number of deaths $\left(y_{t}\right)$ and the number of samples tested $\left(z_{t}\right)$, while the same for $z_{t}$ comes from $x_{t}$ and $y_{t}$. We observe a different scenario for $y_{t}$, where the variance in errors solely depends on itself. Thus, from Figure 2 we can describe a weak causal relationship for our three time series, where $x_{t}$ and $y_{t}$ cause $z_{t}, y_{t}$ and $z_{t}$ cause $x_{t}$ and $y_{t}$ causes itself. We establish that SVAR modelling of the three time series is significant. Please refer to the online appendix for the estimates of various parameters.

\section{B. What do we know about the Impulse Responses?}

One way to interpret the results of SVAR is to see the impulse response functions. In this section we talk about the responses in number of confirmed cases when an impulse is injected to the testing numbers. The other impulse responses can be found in the online appendix. News articles 22 1] report that more than $75 \%$ of the confirmed cases are asymptomatic which can only be de-

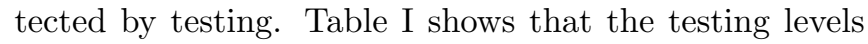
in India are very low compared to the Western countries, and even though we see a gradual increase in $z_{t}$ over time in Figure1, this is not enough. This raises concerns over the actual number of COVID-19 cases in the country which might be higher than the confirmed cases reported by relevant agencies.

We discuss the impact of doubling the testing capacity for

\footnotetext{
${ }^{1}$ The sensitivity analysis for the off-diagonal elements of the A matrix showed that $a_{1,3}$ and $a_{3,1}$ are not sensitive to other values while the other elements were highly sensitive to the other estimated parameters
}

two cases. In the first case, we do it for a single day, while in the second case, we maintain the increased testing for a prolonged period. The first case results in an increase of $9.8 \%$ in confirmed cases for COVID-19 contemporaneously which goes back to zero in about a week's time. The second case leads to a steady rise of $13.84 \%$ in confirmed COVID-19 cases for a week and then becomes constant at that level. This can be seen in Figure 3. Increased testing is evidently is beneficial, as we are then able to identify and treat more cases earlier. This also helps in curbing the spread of the disease as accruing cases are isolated from the general population, thus bringing down the $R_{0}$ value, the details of which are in Section VII

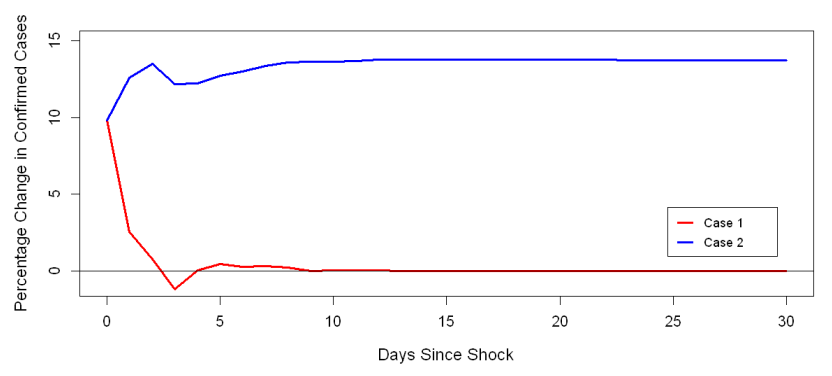

FIG. 3. Impulse response functions for the two cases described

\section{METEOROLOGICAL FACTORS}

\section{Do Temperature and Humidity affect the number of COVID-19 cases?}

There is variation in factors like temperature and humidity over geographies and also over time since January 30 , which was when the first case was detected. These meteorological factors are exogenous with their own trend in nature. We want to look at the effects that this may or may not have on the number of cases in India, and so we use fixed effects model, thus taking into account heterogeneity at city level and getting the relationship with temperature, humidity and time nationally.

Studies in different places (Bashir et al. 2020 in New York, Qi et al. 2020 in China and Şahin 2020 in Turkey) have found significant relationships between the number of COVID-19 cases and meteorological factors. We conduct a study for five cities- Indore, Ahmedabad, Delhi, Jaipur and Mumba: ${ }^{2}$. These have different geographical features, climate and high number of confirmed

\footnotetext{
2Indore: $S D_{T e m p}=2.051$ (Standard Deviation of Temperature), $S D_{R H}=8.261$ (Standard Deviation of Relative Humidity) Ahmedabad: $S D_{\text {Temp }}=1.819, S D_{R H}=8.283$

Delhi: $S D_{T e m p}=3.737, S D_{R H}=8.164$

Jaipur: $S D_{T e m p}=3.478, S D_{R H}=9.316$

Mumbai: $S D_{\text {Temp }}=1.497, S D_{R H}=6.107$
} 
COVID-19 cases. We check for effect of Temperature and Relative Humidity on the same. We observe some degree of correlation, and lag of 3 for moving average values of temperature and humidity (window size of 4 (including contemporaneous point and 3 previous days' values)) is taken for this analysis. In addition, a linear and quadratic time trend is included.

$$
\log \left(c_{i t}\right)=\alpha_{i}+\beta_{1} t+\beta_{2} t^{2}+\beta_{3} \cdot \text { Temp }_{i t}+\beta_{4} \cdot R H_{i t}+\epsilon_{i t}
$$

In the above equation, $i$ is for a city and $\alpha_{i}$ is the fixed effect of that city. $c_{i t}$ is the number of confirmed COVID19 cases, $T e m p_{i t}$ is moving average of temperature over four days, and $R H_{i t}$ is moving average of Relative $\mathrm{Hu}-$ midity over four days. The significance of coefficients is tabulated in Table III. The fixed effects of each city can be found in online appendix

\begin{tabular}{|c|c|c|c|c|}
\hline Coefficient & Estimate & Std. Error & t value & $\operatorname{Pr}(>|t|)$ \\
\hline \hline$\beta_{1}(t)$ & 0.2076 & 0.0151 & 13.7819 & $<0.001 * * *$ \\
$\beta_{2}\left(t^{2}\right)$ & -0.0020 & 0.0002 & -8.8063 & $<0.001 * * *$ \\
$\beta_{3}\left(\right.$ Temp $\left._{i t}\right)$ & -0.1783 & 0.0520 & -3.4288 & $<0.001 * * *$ \\
$\beta_{4}\left(R H_{i t}\right)$ & -0.0188 & 0.0132 & -1.4221 & 0.1561 \\
\hline
\end{tabular}

TABLE III. Fixed Effects Model estimates for $\log \left(c_{i t}\right)$

Results: Table III shows a significant coefficient for moving average of temperature, which is negative. This is in coherence with the other studies which had found temperature to be negatively significant in different places. As we have controlled for time, we conjecture that the expected number of COVID-19 cases in a city with higher temperature will be lower than the expected number of cases in another city with a lower temperature on the same day, provided the fixed effects of these two cities are insignificantly different. We fail to find any significant relationship with Relative Humidity.

\section{EXPONENTIAL SMOOTHING}

Similar to regression, exponential smoothing is a live forecasting technique. In regression, we permit for a finite lag, whereas here, all weighted past data points are utilised. There is a clear time trend in data albeit no seasonality, so Holt smoothing is implemented.

Forecast equation: $\hat{y}_{t+h \mid t}=l_{t}+h b_{t}$

Level equation: $l_{t}=\alpha y_{t}+(1-\alpha)\left(l_{t-1}+b_{t-1}\right)$

Trend equation: $b_{t}=\beta\left(l_{t}-l_{t-1}\right)+(1-\beta) b_{t-1}$

Here, $l_{t}$ is the estimate of level of series at time $t, b_{t}$ is an estimate of trend (slope) of series at time $t, \alpha$ is the smoothing parameter of the level, $0<\alpha<1, \beta$ is the smoothing parameter of trend, $0<\beta<1$.

Forecasting exercise is performed, where the results are updated every 10 days beginning from March 12, and forecasts and actual numbers are plotted in Figure 4

Observational proposition: The forecast values here give a lower bound on cases, and actual numbers top the predicted trend based on past values in general. So when the actual numbers begin meeting the forecast or are less than the latter, we can say that the prior trend has exhausted and as a result, we are either at the peak of number of cases, or the downturn in number of cases is expected in days to come.

Similar proposition holds for death statistics, the details of which can be found on the online appendix.

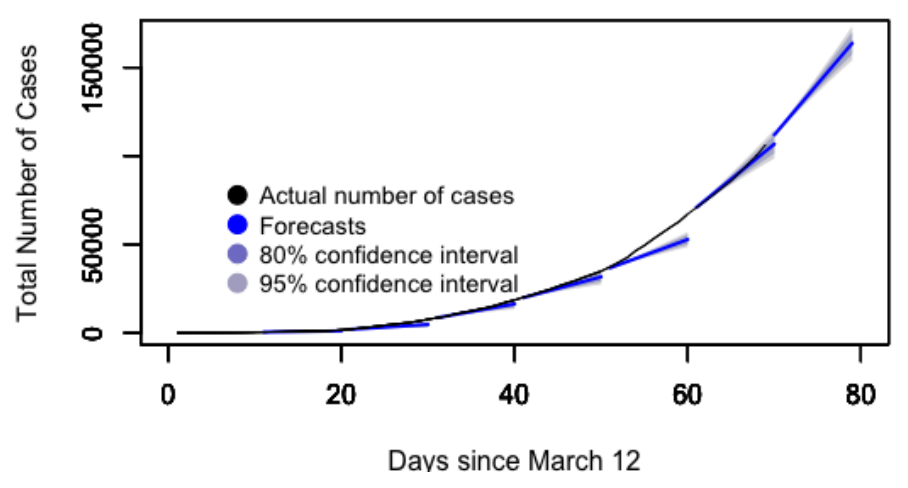

FIG. 4. Forecasting exercise

\section{COMPARTMENTAL MODELLING}

\section{A. How does length of lockdown change disease dynamics?}

Classical SEIR: We employ a deterministic Susceptible Exposed Infectious Recovered (SEIR) model based on progression of disease, epidemiological status of individuals and intervention measures of disease transmission. Figure 6 depicts the model structure, and differential equations below are solved to get solutions for certain cases that are plotted in Figure 5 .

$\frac{d S}{d t}=\frac{-\beta I S}{N}, \frac{d E}{d t}=\frac{\beta I S}{N}-a E, \frac{d I}{d t}=a E-\gamma I, \frac{d R}{d t}=\gamma I$

$S+E+I+R=N, N$ is the total population.

Assumptions:

1. Total population is constant, natural birth and death rates neglected, no new individuals travel in.

2. Latent and infectious periods of the virus are exponentially distributed.

3. Infectious individuals based on probabilistic process either recover or die and become removed class, and no more participate in the SEIR chain.

4. Population is homogeneously well mixed.

5. Every individual belongs to one of the classes.

An important concept here is that of reproduction number, $R_{o}=\frac{\beta}{\gamma}$. This number captures the dynamics 

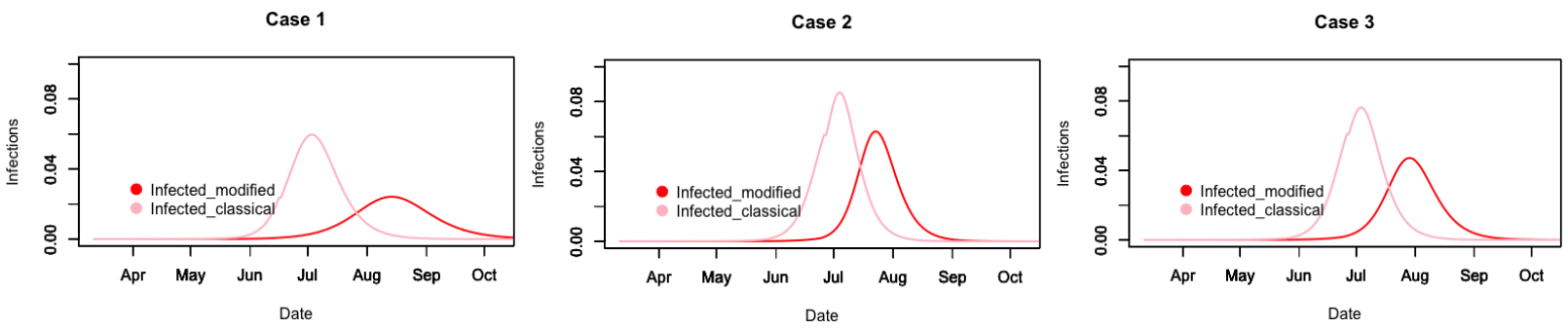

FIG. 5. Plots of case-wise analysis of SEIR modelling, classical and modified, y-axis is as a fraction of the whole population

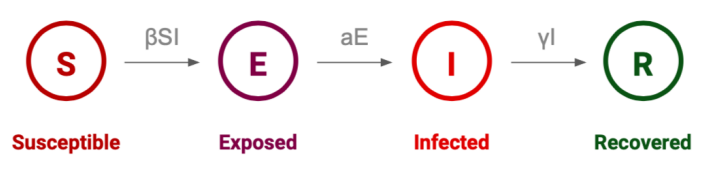

FIG. 6. Population compartments \& model parameters

of disease spread in the population. A strong assumption is for it to be a constant for the country, as it would differ because of varying geographies and demographics, but that is how we have done it in this study. Estimates of $R_{o}$ are a potential way of looking at impact and extent of any policy implementation like lockdown.

Constant parameters in the model [13]:

$a=\frac{1}{\text { latent_period }}=\frac{1}{5.2}, \gamma=\frac{1}{\text { infectious_period }}=\frac{1}{2.3}$

Three cases have been considered for plots:

Case I: The partial lockdown, as it is right now, is extended indefinitely (with an $R_{o}$ value of 2.2). Initial value of $R_{o}$ is taken to be 4 , and lockdown period $R_{o}$ is 1.45 , based on fitting actual data points for different periods of time for India, and then corroborating the estimate with other studies mentioned in Section II.

Case II: Partial lockdown ends by end of June, and everything reopens to near normalcy.

Case III: Partial lockdown opens to a more conservative normalcy post June (work from home and online classes wherever possible, restricted travel services).

$68.84 \%$ population is rural and $31.16 \%$ is urban [6]. $28.6 \%$ of rural and $23.6 \%$ of the urban population is below 14 years of age. As on May 24, Maharashtra, Tamil Nadu, Gujarat and Delhi account for $67 \%$ of the total number of cases reported in country. Goa, Dadra \& Nagar Haveli, Arunachal Pradesh, Mizoram, Lakshadweep, Nagaland, and Sikkim had one/very few cases that recovered, so we remove these states and UTs from our analysis, which make up $0.52 \%$ of the population. Also, we consider urban and $30 \%$ of rural population in the analysis.

\section{B. How do we account for population heterogeneity?}

Age structured SEIR: The structuring of model can be done in several ways by including age based or geo spa- tial data, or a combination of multiple, thus removing assumption (4). We have picked and demonstrated age structured compartmental model in this paper. Figure 7 presents the altered model, where we partition the compartments and solve the listed differential equations. The two partitions $i$ and $j$ are taken to be senior citizens $(\geq 60$ years age, comprising $8.5 \%$ of the total population), and everyone else respectively. We have demonstrated one case in Figure 8, where the lockdown with relaxations ends by end of June, and restrictive normalcy follows.

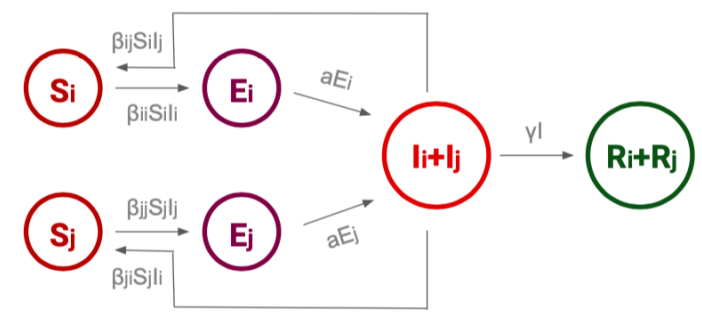

FIG. 7. SEIR accommodating two age groups $j$ and $i$

$$
\begin{gathered}
\frac{d S_{i}}{d t}=-\frac{\lambda_{i} S_{i}}{N}, \frac{d S_{j}}{d t}=-\frac{\lambda_{j} S_{j}}{N}, \frac{d E_{i}}{d t}=\frac{\lambda_{i} S_{i}}{N}-a E_{i}, \\
\frac{d E_{j}}{d t}=\frac{\lambda_{j} S_{j}}{N}-a E_{j}, \frac{d I_{i}}{d t}=a E_{i}-\gamma I_{i}, \frac{d I_{j}}{d t}=a E_{j}-\gamma I_{j} \\
\frac{d R_{i}}{d t}=\gamma I_{i}, \frac{d R_{j}}{d t}=\gamma I_{j}
\end{gathered}
$$

$\lambda$ denotes the age specific force of infection, and $\lambda_{j}=\beta_{j j} I_{j}+\beta_{j i} I_{i}, \lambda_{i}=\beta_{i j} I_{j}+\beta_{i i} I_{i}$

We see that while cases continue to rise, unplanned nor-

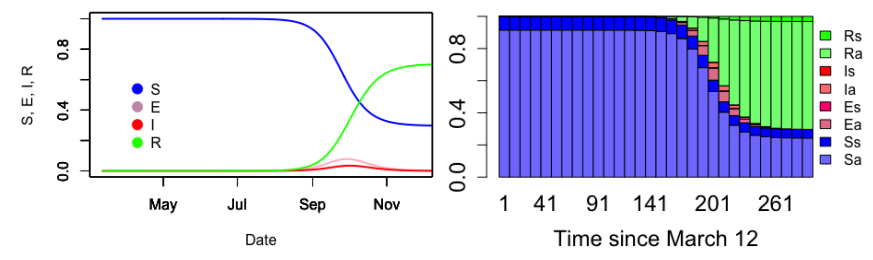

FIG. 8. Results of SEIR model, a) time based chart, b) chart showing diseased population for both sections, $i$ and $j$ 
malcy can lead to an increase in number of cases, thus rendering the lockdown futile. But we may expect effective contact tracing and quarantining of cases as we are better prepared to deal with the problem now.

\section{Can we do better than classical assumptions?}

We do away with assumption (5).

Proposition: For a disease, the parameters $\gamma, a, \lambda$ are a constant. But since the declared pandemic has caused countries to impose lockdowns, the contact pattern and hence $\beta$ alters. The effect of lockdown is reflected in not just changing $\beta$ in the model, but also in changing total level of susceptible population (which is S). This is also the policy implication- lockdown is meant to decrease contact and total population susceptible to the disease.

Continuing from classical model assumptions, we consider $10 \%$ of the rural population and urban aged above 14 during lockdown. For partial lockdown, we consider $80 \%$ urban and $20 \%$ rural aged above 14 . For restrictive normalcy, we consider $85 \%$ urban and $20 \%$ rural population. Based on this model, we present case wise analysis in Figure 5 alongside classical SEIR.

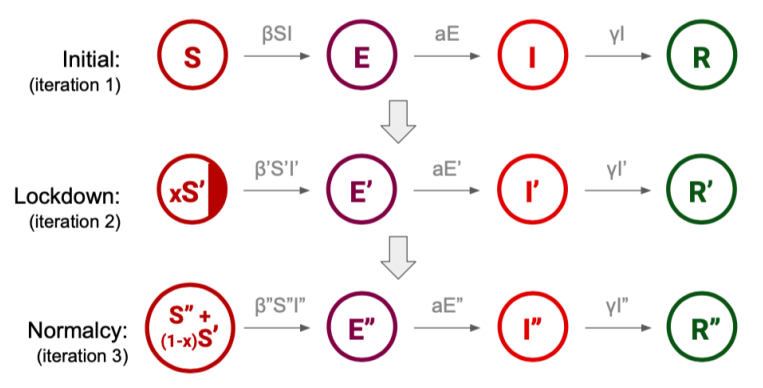

FIG. 9. Evolving compartment SEIR

\section{Takeaways from SEIR}

1. By plotting Figure 5, we study the case wise scenario, but we also look at the effect that assumptions has on the model as a whole. The results are always sensitive to the model assumptions, so we need to be careful before we accept or discard the forecasts of any model.

2. The initial reduction in susceptible population due to timely imposed lockdown has brought down the number of cases because of a complete graph shift in the evolving compartment SEIR, as depicted in the online appendix

3. The curve from modified SEIR is flatter and delayed because by reducing susceptible population by multiple iterations, we have essentially bettered the societal response. As the severity of lockdown increases, the gap between modified and classical model peak also increases.
4. The total susceptible population is not zero, nor are the recoveries equal to 1 . So although it is hard to comment on what we can anticipate, but even after the peak and downturn, virus does not die out.

5. Since actual data has not been worked with in this modelling, model fit or forecast sensitivity is not applicable in the conventional sense.

6. Relaxing lockdown anytime after peak does not cause the resurgence in number of cases, but premature opening results in a spike at the peak.

\section{FORECASTS AND ACTUAL VALUES}

We present 5-day (not more, because both regression and exponential smoothing are short term forecasting methods, SEIR producing long term predictions) forecasts for the number of confirmed COVID-19 cases from different models in this paper. While regression provides an upper bound on number of cases, exponential smoothing gives linear output (here, 5757) which is a lower bound. Together they provide an interval of expected number of cases.

\begin{tabular}{|l|l|l|l|}
\hline Date & VAR & SEIR & Actual \\
\hline \hline May 21 & 6204 & 6164 & 6023 \\
May 22 & 6208 & 6628 & 6536 \\
May 23 & 6879 & 6831 & 6665 \\
May 24 & 7064 & 7210 & 7111 \\
May 25 & 7346 & 7532 & 6414 \\
\hline
\end{tabular}

TABLE IV. 5-day forecasts from models

\section{CONCLUSION}

The study helps in capturing the quantifiable idea of level of under reporting. Doubling testing rates leads to detection of $13.84 \%$ more COVID-19 cases. Tests do not affect the death statistics, the variation of which is not captured in this methodology, for instance its dependence on level of healthcare available or virus mutation. Temperature negatively affects the number of cases in a city, while humidity's effect is statistically insignificant.

Exponential smoothing is a fairly simplistic technique that captures the trend, which is used for identifying change in trend- which happens at the peak or in the downturn in number of cases. We construct epidemiological models and see that the results from mathematical modelling are highly sensitive and specific to the choice of model and its underlying assumptions. This is used to study lockdown scenarios and a clear trade off between peak value of number of cases and number of days to the peak is observed. From the classical model, premature opening of lockdown post June can result in over $44.06 \%$ more peak cases as compared to an extended lockdown scenario. We can model better with more data about demographic contact rates and lockdown effects, which have been evaluated with ballpark figures. 
Since the study is based on reported statistics, it does not account for virus properties and heterogeneity in time series because of its specific and geo spatial variations. Short term models help in efficient planning for testing and healthcare facilities, while long term predictions are the key to implementation of policies like lockdown and making resources available to the general population, a large proportion of which cannot work from home and depends on daily to monthly earnings. By studying both, the study tries to capture entire picture of the pandemic. The nation must brace itself for a long and hard battle, for every case and death is more than just a statistic and it is crucial to come together to prevent losses.

\section{CONFLICTS OF INTEREST}

We declare that we have no conflicts of interest.

\section{FUNDING}

This research received no specific grant from any funding agency in the public, commercial, or not-for-profit sectors.
[1] $76 \%$ covid-19 cases in karnataka asymptomatic. https://www.thehindu.com/news/national/karnataka/ over-76-of-covid-19-patients-in-karnataka-areasymptomatic/article31546496.ece

[2] $80 \%$ covid-19 cases asymptomatic. //economictimes.indiatimes.com/industry/ healthcare/biotech/healthcare/no-symptoms-in80-of-covid-cases-raise-concerns/articleshow/ 75260387.cms? from $=\mathrm{mdr}$

[3] (2019). https://www.populationpyramid.net/india/ $2019 /$

[4] (2020). https://www.worldometers.info

[5] (2020). https://www.covid19india.org.

[6] (2020). Census. https://censusindia.gov.in

[7] (2020). Covid-19 data repository by the center for systems science and engineering (csse) at johns hopkins university. hhttps://github.com/CSSEGISandData/COVID19 .

[8] (2020). Ministry of health and family welfare, india. https://mohfw.gov.in.

[9] (2020). World weather online. https: //www.worldweatheronline.com

[10] Bashir, M. F., Ma, B., Bilal, Komal, B., Bashir, M. A., Tan, D., and Bashir, M. (2020). Correlation between climate indicators and covid-19 pandemic in new york, usa. Science of The Total Environment, 728:138835.

[11] Deb, S. and Majumdar, M. (2020). A time series method to analyze incidence pattern and estimate reproduction number of covid-19.

[12] Gupta, Rajan, Pal, and Kumar, S. (2020). Trend analysis and forecasting of covid-19 outbreak in india. medRxiv.
[13] Hamzah, F. A. B., Lau, C. H., Nazri, H., Ligot, D. V., Lee, G., Cheng Liang Tan, Mohammad Khursani Bin Mohd Shaib, U. H. B. Z., Abdullah, A. B., Chung, M. H., Ong, C. H., Chew, P. Y., and Salunga, R. E. (2020). Coronatracker: World- wide covid-19 outbreak data analysis and prediction. [Preprint]. World Health Organisation Bulletin.

[14] Petropoulos, F. and Makridakis, S. (2020). Forecasting the novel coronavirus covid-19. PLoS ONE 15(3).

[15] Prem, K., Cook, A. R., and Jit, M. (2017). Projecting social contact matrices in 152 countries using contact surveys and demographic data. PLoS Comput Biol 13(9).

[16] Qi, H., Xiao, S., Shi, R., Ward, M. P., Chen, Y., Tu, W., Su, Q., Wang, W., Wang, X., and Zhang, Z. (2020). Covid-19 transmission in mainland china is associated with temperature and humidity: A time-series analysis. Science of The Total Environment, 728:138778.

[17] Singh, R. and Adhikari, R. (2020). Age-structured impact of social distancing on the covid-19 epidemic in india. arXiv:2003.12055.

[18] Yang, Z., Zeng, Z., Wang, K., Wong, S.-S., Liang, W., Zanin, M., Liu, P., Cao, X., Gao, Z., Mai, Z., Liang, J., Liu, X., Li, S., Li, Y., Ye, F., Guan, W., Yang, Y., Li, F., Luo, S., Xie, Y., Liu, B., Wang, Z., Zhang, S., Wang, Y., Zhong, N., and He, J. (2020). Modified seir and ai prediction of the epidemics trend of covid-19 in china under public health interventions. Journal of Thoracic Disease, 12(3).

[19] Şahin, M. (2020). Impact of weather on covid-19 pandemic in turkey. Science of The Total Environment, $728: 138810$. 